\title{
Improving the data access control using blockchain for
}

\section{healthcare domain [version 1; peer review: 1 approved with}

\section{reservations]}

\author{
Olaosebikan Tahir Yinka1, Su-Cheng Haw (iD), Timothy Tzen Vun Yap (iD1, \\ Samini Subramaniam² \\ ${ }^{1}$ Faculty of Computing \& Informatics, Multimedia University, Cyberjaya, Selangor, 63100, Malaysia \\ ${ }^{2}$ AirAsia Berhad, KLIA2, Selangor, 64000, Malaysia
}

\begin{tabular}{l}
\hline F1 First published: 08 Sep 2021, 10:901 \\
https://doi.org/10.12688/f1000research.72890.1 \\
Second version: 27 Oct 2021, 10:901 \\
https://doi.org/10.12688/f1000research.72890.2 \\
Latest published: 19 Nov 2021, 10:901 \\
https://doi.org/10.12688/f1000research.72890.3 \\
\hline
\end{tabular}

\section{Abstract}

\section{Introduction}

Unauthorized access to data is one of the most significant privacy issues that hinder most industries from adopting big data technologies. Even though specific processes and structures have been put in place to deal with access authorization and identity management for large databases nonetheless, the scalability criteria are far beyond the capabilities of traditional databases. Hence, most researchers are looking into other solutions, such as big data management. Methods

In this paper, we firstly study the strengths and weaknesses of implementing cryptography and blockchain for identity management and authorization control in big data, focusing on the healthcare domain. Subsequently, we propose a decentralized data access and sharing system that preserves privacy to ensure adequate data access management under the blockchain. In addition, we designed a blockchain framework to resolve the decentralized data access and sharing system privacy issues, by implementing a public key infrastructure model, which utilizes a signature cryptography algorithm (elliptic curve and signcryption). Lastly, we compared the proposed blockchain model to previous techniques to see how well it performed.

Results

We evaluated the blockchain on four performance metrics which include throughput, latency, scalability, and security. The proposed blockchain model was tested using a sample of 5000 patients and 500,000 observations. The performance evaluation results further showed that the proposed model achieves higher throughput and lower latency compared to existing approaches when the workload

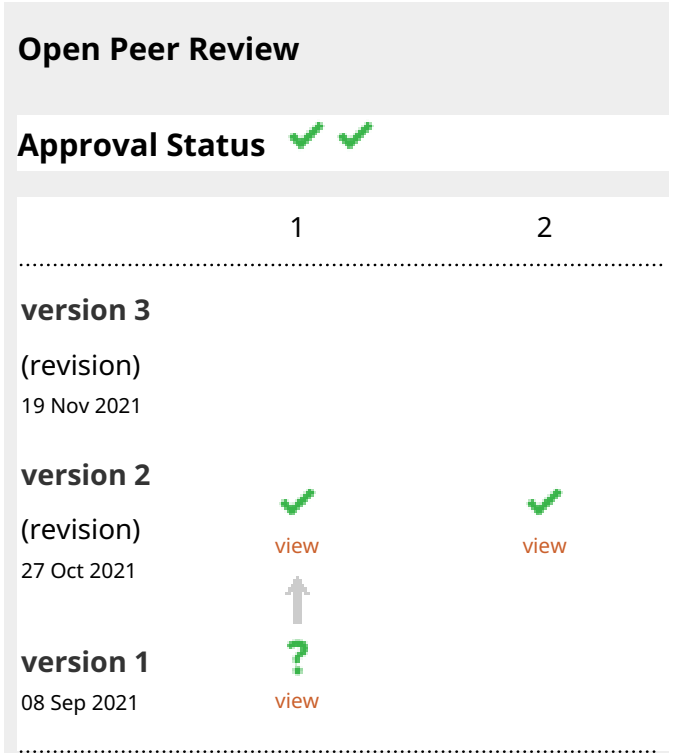

1. Huo Chong Ling, Curtin University Malaysia, Miri, Malaysia

2. Heru Agus Santoso (D), Curtin University Malaysia, Miri, Malaysia

Any reports and responses or comments on the article can be found at the end of the article. 
varies up to 10,000 transactions.

Discussion

This research reviews the importance of blockchains as they provide

infinite possibilities to individuals, companies, and governments.

Keywords

Blockchain, Healthcare datasets, Chaincode, Directed Acyclic Graph, Access Control.

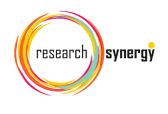

This article is included in the Research Synergy

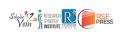

Foundation gateway.

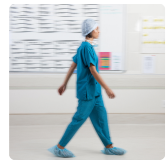

This article is included in the Health Services

gateway.

Corresponding author: Su-Cheng Haw (sucheng@mmu.edu.my)

Author roles: Tahir Yinka O: Conceptualization, Formal Analysis, Investigation, Methodology, Validation, Writing - Original Draft Preparation; Haw SC: Project Administration, Supervision, Writing - Review \& Editing; Yap TTV: Supervision, Writing - Review \& Editing; Subramaniam S: Writing - Review \& Editing

Competing interests: No competing interests were disclosed.

Grant information: The author(s) declared that no grants were involved in supporting this work.

Copyright: @ 2021 Tahir Yinka O et al. This is an open access article distributed under the terms of the Creative Commons Attribution License, which permits unrestricted use, distribution, and reproduction in any medium, provided the original work is properly cited.

How to cite this article: Tahir Yinka O, Haw SC, Yap TTV and Subramaniam S. Improving the data access control using blockchain for healthcare domain [version 1; peer review: 1 approved with reservations] F1000Research 2021, 10:901

https://doi.org/10.12688/f1000research.72890.1

First published: 08 Sep 2021, 10:901 https://doi.org/10.12688/f1000research.72890.1 


\section{Introduction}

The US social insurance system, such as healthcare, is gradually obtaining digital medical information, thanks to the fastexpanding availability of data which will considerably build up the number of medical information collected securely. ${ }^{1}$ Furthermore, a change in perspective in the medical services sector has seen the new trend of computerizing medical records. The medical services sector is therefore rapidly rising in data volume as far as randomness and simplicity are concerned. Although the public healthcare sector is struggling with the magnitude of big data, security and privacy concerns are converging as threats and vulnerabilities grow. ${ }^{2,3}$

The modern and revolutionary technology to build safer, more private, and interoperable health systems, blockchain technology has proven to be efficient. The aim of this paper is to propose a decentralized model for sharing and storing medical information utilizing blockchain technology to ensure security, preserving complete privacy, and allowing the owner to be completely in control of their data. In this research, additional efforts were made to harness and itemize all blockchain technology technical tools and the current influence of this developing technology on healthcare and industry.

Recent studies in the healthcare sector dealing with is-sues of scalability have used blockchain. Nonetheless, the application of blockchain in these studies was limited to the healthcare dataset index and the storage of verified transaction by endorsing peers. In contrast to these approaches, the current blockchain model pro-poses the storage of all medical datasets in blockchain and also improving data throughput limitations. In light of these proposed improvements, Yang et al. ${ }^{4}$ proposed the use of blockchain as an index of a medical dataset and a list of user's information. Similarly, Daraghmi et al. ${ }^{5}$ introduced a data-sharing and access authentication scheme based on a private blockchain. Likewise, Genestier et $a l^{6}{ }^{6}$ offered a novel proposal for reshaping healthcare permission monitoring using blockchain technology and allow users to access their entire health record data. In their implementation, there is no permission settings and no access restriction. MedRec ${ }^{7}$ is a software system developed using blockchain technology by the MIT media lab to handle electronic health records. They created a hospital management system that responds to four major issues: disjointed, delayed access to health care information; process compatibility; respect for autonomy; and enhanced data quantity and quality for clinical research.

Medical Chain ${ }^{8}$ project began in 2016, with the launch of the first model in mid-2017. Later in 2017, a partnership with the Linux Foundation was created. In the early months of 2018, a beta version of the program was published. The primary objective is to develop a single, accurate version of the user's health data.

MedBlock, ${ }^{9}$ which is architecture based on a hybrid blockchain to protect Electronic Medical Records (EMRs). Their design nodes identify them as endorsers, orderers, and committers. Yet, the access control strategy to allow third-party researchers access to medical data is not specified precisely.

Xia et al. ${ }^{10}$ introduced the Blockchain-Based Data Sharing (BBDS) system allowing owners and participants to access EMR from a shared repository theory based on positive identity and key verification.

Diversely, other research projects related to adopting blockchain in the healthcare domain are summarized as follows.

Blockchain Health, ${ }^{11}$ ASTRI ${ }^{12}$ and Bloq $^{13}$ allow users to use the integrated platform to share health data with researchers, which creates a tamper-proof knowledge custody chain. Similarly, HIE of One ${ }^{14-16}$ is a blockchain software initiative that provides resources for patients to access their own records. Hyperledger ${ }^{17}$ and IBM Blockchain ${ }^{18}$ is hyperledger fabric's first regulated platform, allowing the development of company blockchain platforms that users can monitor and spread through various organizations.

All the principal functions of blockchain technology and some true blockchain applications in healthcare were discussed at this point. More concretely, issues addressed in this chapter include authentication management, identity management, and cryptography. In the next section we will clarify the model that helps to il-lustrate how the blockchain network communicates with the developed model.

The paper contributions are summarized as follows:

1. A file storage and sharing system for a pure decentralized network utilizing blockchain technology is proposed.

2. An extensive evaluation of the proposed system, which demonstrated that our proposed system achieves higher throughput and lower latency com-pared to some existing approaches at higher work-load varied up to 10,000 transactions. 


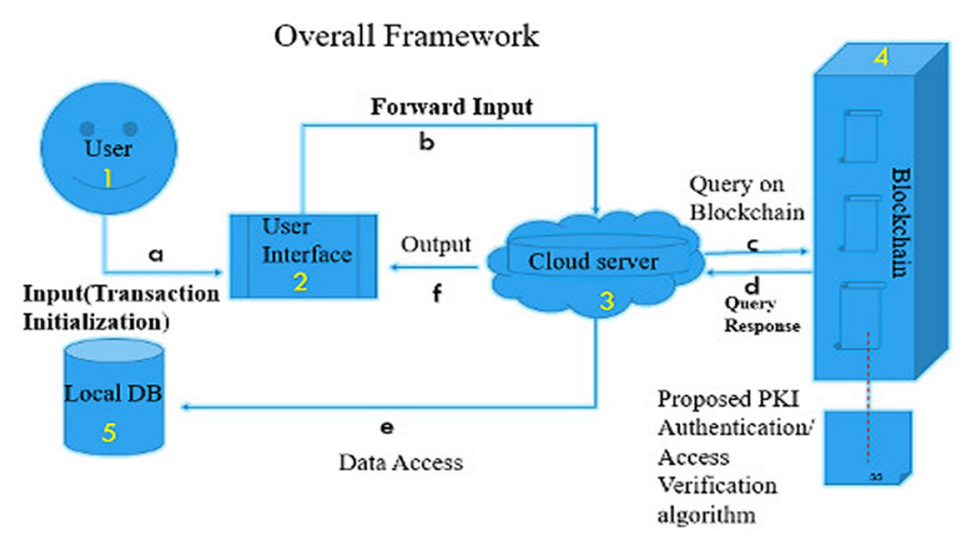

\section{Figure 1. Overall framework of proposed system.}

\section{Methods}

The overall data storage workflow is displayed in Figure 1 based on the following steps:

(1) A user joins through the graphical user inter-face (GUI) and the request for permission to access information can be inserted into the network when successfully authenticated.

(2) The user's request is sent to the cloud server by the GUI.

(3) The blockchain component, the server query enabled, or revoked access.

(4) The blockchain component handles content verification, signing, and encryption via Public Key Infrastructure (PKI).

(5) Finally, data updated/stored with the authenticated token takes place.

From Figure 1, steps (a) to (f) depict the procedures for accessing a data source:

(a) A user authenticates using the GUI. Upon successful authentication, user input will be taken into the system.

(b) Through the GUI, user input is forwarded to the cloud server.

(c) The cloud server then run queries against the blockchain for the user's access token by running the proposed PKI algorithm.

(d) The access token retrieved is been decrypted with the appropriate user keys.

(e) Data access is granted and retrieved with the help of a database connector.

(f) The server returns updates to display data in a format that is readable to the user.

\section{Case study: implementing the proposed model prototype to healthcare}

We first analyzed a case study concerning the healthcare domain. We will then describe each of the key components in the case studies: (i) join function, (ii) content authentication function, and (iii) secure content access function.

The whole scheme works as follows. Consider a healthcare scenario of file storing and sharing. For each transaction, the 'patient' provides the contents to be stored and accessed. The 'hospital administrator' and 'doctor' request access to these contents. We assume that 'hospital administrator' and 'doctor' use our proposed model (see Figure 1), acting as the search mechanism to engage in the process of searching for content. 


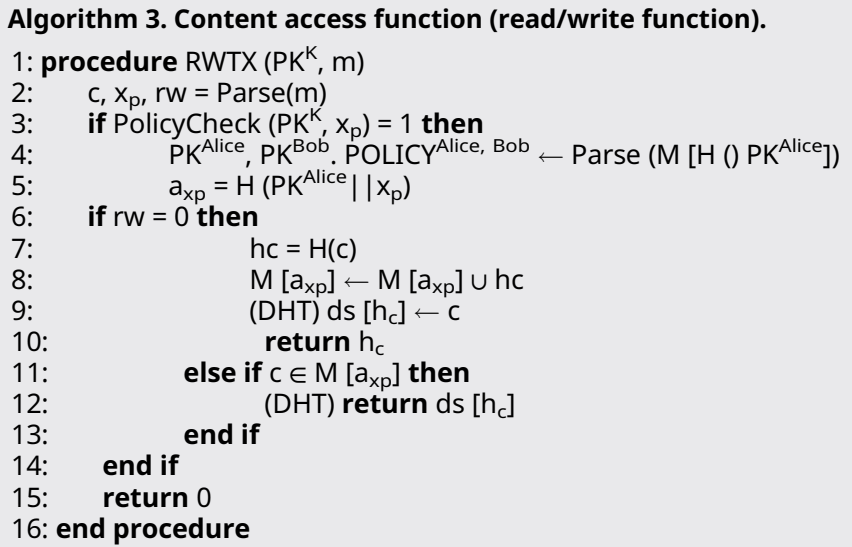

in an asynchronous manner. The number of requests is set from 1000 to 10,000 transactions, with increments of 1000 transactions in-between them, a sample digitized data collection, namely OpenMRS that consists of 5000 patients and 500,000 observations is used for our evaluation (see Table 1). Our proposed approach was benchmarked against approaches proposed by $^{4,5}$ for fair comparison.

\section{Case study: implementing the proposed model prototype to healthcare}

Figure 2 depict the average latency across specific block sizes, analyzing various datasets or arrival rates of transactions. From Figure 2, we made several observations. This include the average latency in our approach is almost two-times less than that of average latency in both approaches by ${ }^{4,5}$ for all block sizes, except for when the transaction is at $1000 \mathrm{TX}$, we saw that latency of all approaches are lower. This is because the volume of pending transactions at validation stage grew on high count which affected commit latency.

\section{Evaluating the impact of endorsement policy}

Figure 3 depict the average latency time of endorsement policy with the various number of transactions per second (TPS) (for the number of transactions ranging from 1000 to 10000). From Figure 3, it can be observed that the number of subpolicies and the number of signature validation has caused high latency for the join, content authentication and secure content access functions in ${ }^{4,5}$ approaches.

Impact of state database

We study the throughput and latency over varying transaction arrival rates for database utilized in the three approaches with different transaction complexity.

From Figures 4 and 5, shows that as transaction arrival increased for read/write only complexity, the throughput increased and latency decreased. This was observed while utilizing CouchDB as the state database.

The result shows that GoLevelDB performance was better than CouchDB with respect to ledger database. The reason behind the relatively good performance is the existence of key-value state database embedded in the peer mechanism.

Table 1. Experiment parameters.

\begin{tabular}{|l|l|}
\hline Evaluation parameters & Evaluation values \\
\hline Transactions/Queries submitted & $1000,2000,300,4000,5000,6000,7000,8000,9000$ and 10,000 \\
\hline Block sizes & $200,400,600$ \\
\hline Transaction in byte & $1,000-10,000$ \\
\hline The stored health records & $10,000-100,000$ \\
\hline
\end{tabular}




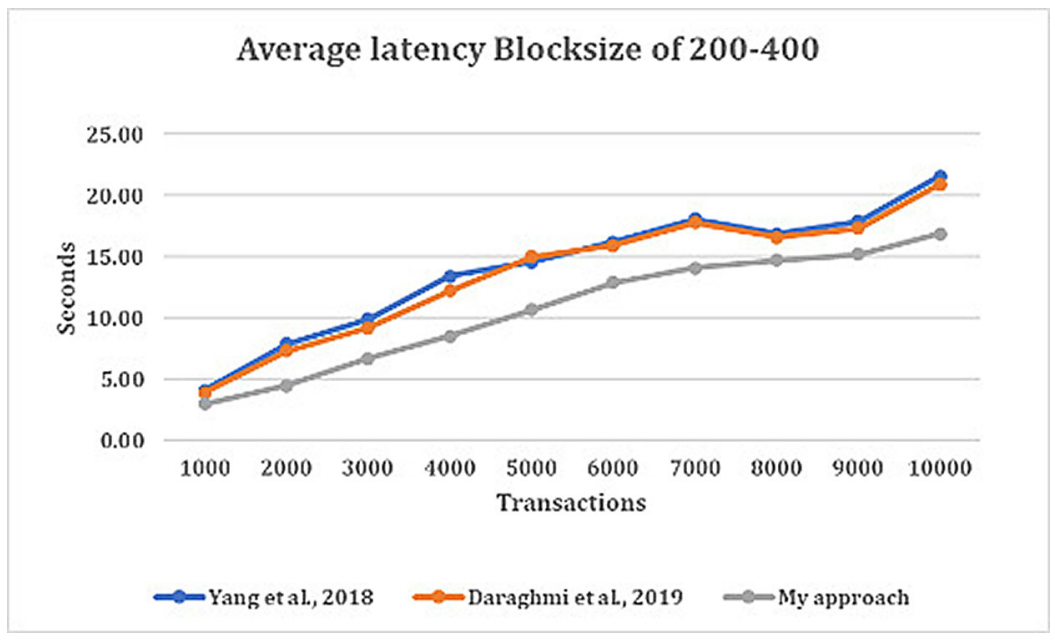

Figure 2. Average latency with block size of $200-400$ on various transaction arrival rates.

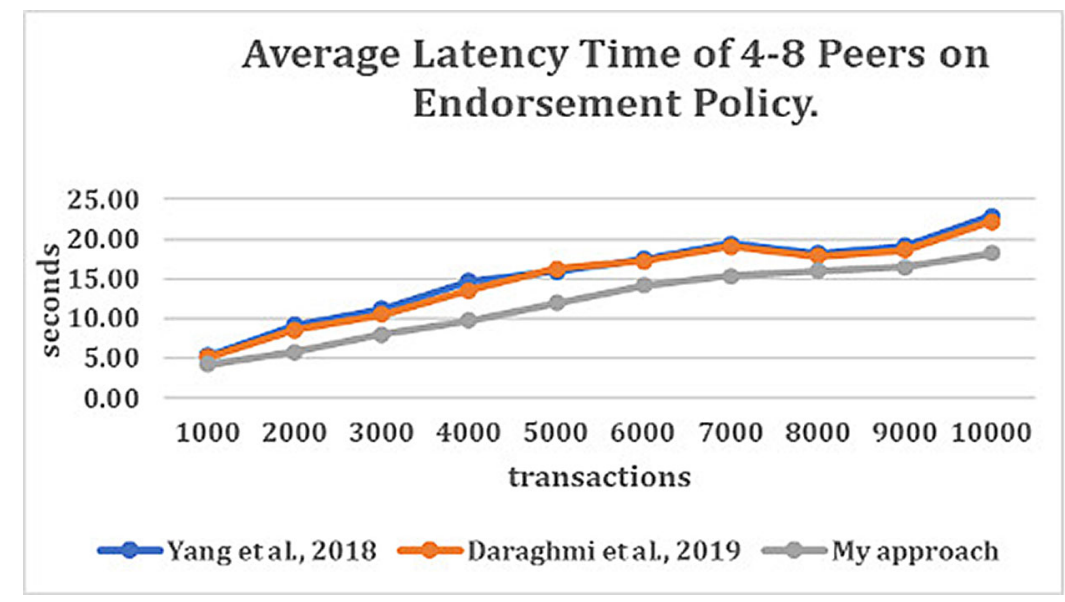

Figure 3. Average latency time of 4-8 peers on endorsement policy.

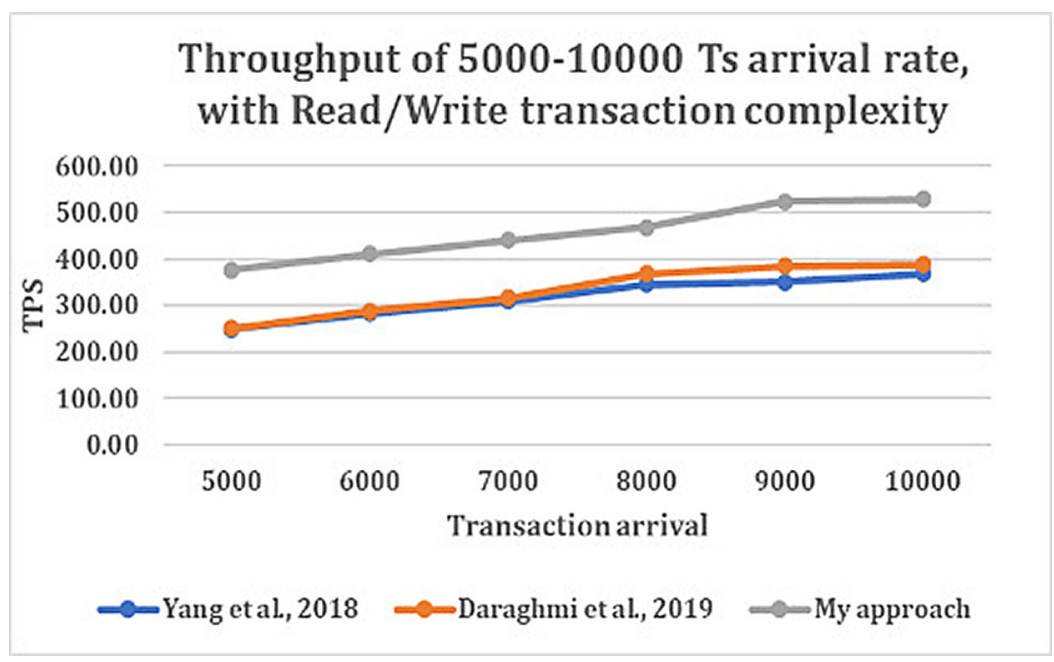

Figure 4. Throughput of 5000-10000 transaction arrival rate with read/write transaction. 


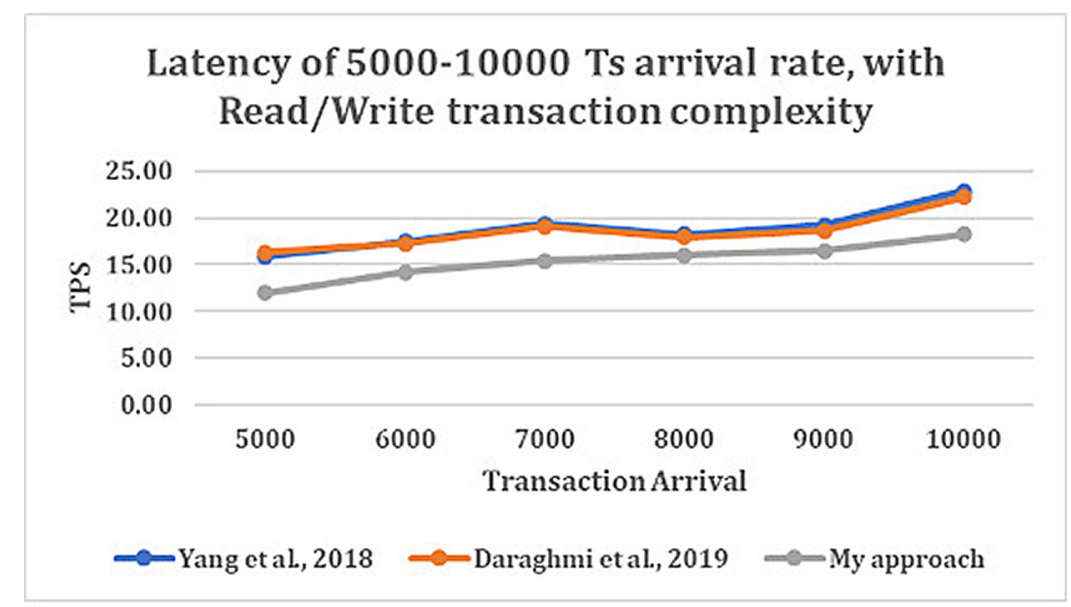

Figure 5. Latency of 5000-10000 transaction arrival rate with read/write transaction.

\section{Discussion}

The performance evaluation results further showed that the proposed model achieves higher throughput and lower latency compared to existing approaches when the workload varies up to 10,000 transactions.

It's also worth noting that the proposed model preserves confidentiality and security by controlling transactions with timed-based chaincodes. The use of sophisticated encryption maintains security and access control.

Security and access control are maintained by the adoption of advanced encryption (such as the proposed hybrid PKI) and authentication methods that defend against adversarial efforts to change unwanted entry when issuing encryption information to verify and validate transactions to network users.

\section{Conclusions}

We had presented a model, which describes the interaction between a blockchain network and the designed model. To ensure a safe and private environment to communicate and store health data, the designed model exploits the most significant elements of blockchain technology while suppressing features that are not significant. In future work, we expect to perform refined simulations so that our model is tested more rigorously. We could do this by downloading and testing a wider range of various blockchain frameworks in a verified setting, for instance, using the blockchain instance of Amazon Web Services' blockchain.

\section{Author contributions}

Olaosebikan Tahir Yinka constructed the protytpe and conducted the experimental evaluations under the guidance of his supervisors, Su-Cheng Haw and Timothy Tzen Vun Yap. Samini Subramaniam is the external collaborator while $\mathrm{Su}-\mathrm{Cheng}$ Haw is the corresponding author for this paper.

\section{Data availability}

No data are associated with this article.

\section{Software availability}

- Source code available from: https://github.com/captaintaheer/Solution_Code

- Archived source code at time of publication: https://doi.org/10.5281/zenodo.5226652

- License: Data are available under the terms of the Creative Commons Attribution 4.0 International license (CCBY 4.0).

\section{Ethics approval}

Ethical approval was given by the ethical approval board of Multimedia University, with number: EA1762021. 
1. Zyskind G, Nathan O, Pentland A: Decentralizing Privacy: Using Blockchain to Protect Personal Data. IEEE Security and Privacy Workshops; 2015.

2. Aljawarneh $S$, Yassein MB, Talafha WA: A resource-efficient encryption algorithm for multimedia big data. Multimedia Tools and Applications. 2015; 76(21): 22703-22724.

Publisher Full Text

3. Hilbert M: Big Data for Development: A Review of Promises and Challenges Development Policy Review. 2015; 34(1), pp. 135-174. Publisher Full Text

4. Yang J, Onik M, Lee N, et al.: Proof-of-Familiarity: A PrivacyPreserved Blockchain Scheme for Collaborative Medical Decision-Making. Appl. Sci. 2019; 1370. Publisher Full Text

5. Daraghmi E-Y, Daraghmi Y-A, Yuan SM: MedChain: A Design of Blockchain-based System for Medical Records Access and Permissions Management. IEEE Access. 2019; 1-1. Publisher Full Text

6. Genestier P: Blockchain for consent management in the eHealth environment: A nugget for privacy and security challenges. J. Int. Soc. Telemedicine eHealth. 2017, April; 5: 1-4.

7. Azaria A, Ekblaw A, Vieira T, et al.: MedRec: Using blockchain for medical data access and permission management. 2nd Int. Conf. Open Big Data. Aug. 2016; (p. 2530).

8. Albeyatti A, Tayeb M, Yin A, et al.: Medicalchain (Whitepaper). 2018; pp. 1-42.

9. Chen M, Qian Y, Chen J, et al.: Privacy Protection and Intrusion Avoidance for Cloudlet based Medical Data Sharing. IEEE Transactions on Cloud Computing. 2016; 1(1). Publisher Full Text

10. Amofa S, Sifah EB, Agyekum KO-B, et al.: A blockchain-based architecture framework for secure sharing of personal health data. IEEE 20th Int. Conf. e-Health Netw., Appl. Services (Healthcom).
$2018 ; 16$.

Publisher Full Tex

11. ASTRI: Whitepaper on Distributed Ledger Technology. 2016. Reference Source

12. Culver K: Blockchain technologies: A whitepaper discussing how the claims process can be improved. Workshop: ONC/NIST Use Blockchain Healthcare Res; 2016.

13. Genestier P: Blockchain for consent management in the eHealth environment: A nugget for privacy and security challenges. J. Int. Soc.Telemedicine eHealth; 2017, April; 5: 1-4.

14. Gropper A, MD: Powering the PhysicianPatient Relationship with HIE of One Blockchain Health IT. H N, S K, H G2017.

15. Distributed ledger technology and blockchain. World Bank. Reference Source

16. T L: Foundation Hyperledger Overview. 2018.

17. Dagher GG, Mohler J, Milojkovic M, et al.: Ancile: Privacypreserving framework for access control and interoperability of electronic health records using blockchain technology. Sustain. Cities Soc. 2018, May; 39: 283297. Publisher Full Text

18. Guo R, Shi H, Zhao Q, et al.: Secure attribute-based signature scheme with multiple authorities for blockchain in electronic healthrecords systems. IEEE Access. 2018; 6 . 1167611686 Publisher Full Text

19. Aazam M, Zeadally S, Harras KA: Health fog for smart healthcare. IEEE Consum Electron Maga. 2020; 9(2): 96-102. Publisher Full Text

20. Shadab A, F AR, S MZHKKR, et al.: Towards Trustworthiness of Electronic Health Record system using Blockchain. Annals of the Romanian Society for Cell Biology. 2021; 25(6): 2425-2434. Reference Source 


\section{Open Peer Review}

\section{Current Peer Review Status: ?}

\section{Version 1}

Reviewer Report 08 October 2021

https://doi.org/10.5256/f1000research.76499.r94763

(c) 2021 Ling $\mathbf{H}$. This is an open access peer review report distributed under the terms of the Creative Commons Attribution License, which permits unrestricted use, distribution, and reproduction in any medium, provided the original work is properly cited.

\section{Huo Chong Ling}

Department of Electrical and Computer Engineering, Curtin University Malaysia, Miri, Malaysia

The authors proposed a decentralized network using blockchain technology to store and share health data securely by adopting a PKI model in their blockchain framework. The PKI model consists of content verification and authentication and secure access. Experiments were conducted to evaluate the performance of the proposed blockchain framework in terms of throughput, latency, scalability, and security, and compared to existing approaches in the literature. The experiments showed promising results, which indicate the feasibility of the framework to be used in the healthcare domain.

However, there are a few doubts regarding the statistical analysis of the experimental results as follows:

1. Do the results obtained consist of the average of several runs or just one run?

2. Are the algorithms used in [4] and [5] implemented as well on the same PC that is used for the proposed approach? This is to ensure fair performance evaluation.

3. The latency results showed the average for the block size of 200-400. How about the latency results for the block size of 400 and above?

Other comments on the article are as follows:

- The year of publication for Yang et al. is 2019, but in your figures, it is shown as 2018.

It's better to write "Proposed approach" instead of "My approach" in the figures.

Reference 20 is not cited in the text.

Is the work clearly and accurately presented and does it cite the current literature? Yes

Is the study design appropriate and is the work technically sound? 
Yes

Are sufficient details of methods and analysis provided to allow replication by others? Yes

If applicable, is the statistical analysis and its interpretation appropriate?

Partly

Are all the source data underlying the results available to ensure full reproducibility?

Yes

Are the conclusions drawn adequately supported by the results?

Yes

Competing Interests: No competing interests were disclosed.

Reviewer Expertise: Security

I confirm that I have read this submission and believe that I have an appropriate level of expertise to confirm that it is of an acceptable scientific standard, however I have significant reservations, as outlined above.

Author Response 22 Oct 2021

Haw Su Cheng.

Do the results obtained consist of the average of several runs or just one run?

Author response: Yes, between 1000 to 10,000 transactions were asynchronously sent to the blockchain server. The number of requests is set from 1000 to 10,000 transactions, with increments of 1000 transactions for every run.

Are the algorithms used in [4] and [5] implemented as well on the same PC that is used for the proposed approach? This is to ensure fair performance evaluation.

Author response: Yes, this was included in the proposed model access chaincodes, which record all 'user' activities, such as the record of shared data or information access history on the blockchain. Algorithm 3 is executed when transaction access is requested, and similarly, Algorithm 4 is executed for read/write transactions.

The latency results showed the average for the block size of 200-400. How about the latency results for the block size of 400 and above?

Author response We have studied latency and throughput up to 600 blocks in the thesis presented for the work. Also, as stated in observations for all block sizes as for all block sizes, except for when the transaction is at 1000, we saw that latency of all approaches are lower.

Other comments on the article are as follows:

The year of publication for Yang et al. is 2019, but in your figures, it is shown as 2018.

Author response: This has been adjusted in figures

It's better to write "Proposed approach" instead of "My approach" in the figures.

Author response: This has been adjusted in figures as advised 
Reference 20 is not cited in the text.

Author response: Citation added.

Competing Interests: No competing interests were disclosed.

The benefits of publishing with F1000Research:

- Your article is published within days, with no editorial bias

- You can publish traditional articles, null/negative results, case reports, data notes and more

- The peer review process is transparent and collaborative

- Your article is indexed in PubMed after passing peer review

- Dedicated customer support at every stage

For pre-submission enquiries, contact research@f1000.com 